\title{
UAW jetzt online melden
}

Patienten können unerwünschte Arzneimittelwirkungen (UAW) jetzt direkt online melden. BfArM und PEI haben die Webseite freigeschaltet.

— Die Zulassungsbehörden BfArM und PEI haben eine Website, auf der Patienten unerwünschte Arzneimittelwirkungen (UAW) oder Impfreaktionen melden können, jetzt testweise freigeschaltet. Damit wolle man europäischen Bestrebungen entgegenkommen, direkten Nebenwirkungsmeldungen durch Patienten mehr Bedeutung einzuräumen, so das BfArM. „Unbekannte Nebenwirkungen könnten gegebenenfalls früher als bisher erkannt und, soweit erforderlich, Maßnahmen zur Risikominimierung eingeleitet werden“, lautet die Begründung der Zulassungsbehörde für das Online-Angebot.

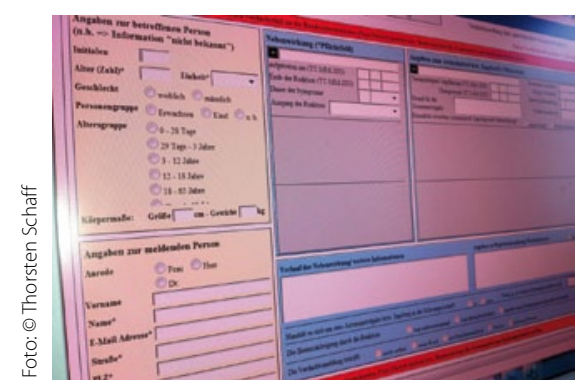

Hier können Patienten UAW melden.

Unter der Adresse www.verbraucheruaw.pei.de können UAWs verschlüsselt den Behörden übermittelt werden. Dabei sind auch persönliche Kontaktdaten anzugeben, die jedoch vertraulich behandelt werden, heißt es. Besucher der Website werden ausdrücklich darauf hingewiesen, „mit möglichen Nebenwirkungen zur weiteren Diagnose und Therapie unverzüglich den behandelnden Arzt zu kontaktieren“.

$\mathrm{CW}$.

\section{Hier muss der Dummy durch eine Anzeige ersetzt werden !!}

